\title{
Extracellular ATP Modulates Calcium Uptake and Transmitter Release at the Neuromuscular Junction
}

\author{
Clark A. Lindgren ${ }^{\mathrm{a}}$ and Dean O. Smith \\ Department of Physiology, University of Wisconsin, Madison, Wisconsin 53706
}

\begin{abstract}
Adenosine, AMP, ADP, and ATP were tested for their ability to modulate evoked quantal transmitter release at excitoropener nerve terminals in the crayfish walking leg. Only ATP was found to have a significant effect, inhibiting release by $43 \%$. To determine whether the effects of extracellular ATP on transmitter release were related to changes in free $\mathrm{Ca}^{2+}$ levels in the nerve terminal, net ${ }^{45} \mathrm{Ca}$ uptake was measured in regions of the nerve near the terminal; although resting ${ }^{45} \mathrm{Ca}$ uptake was increased by $5 \mathrm{~mm}$ exogenous ATP. Thus, extracellular ATP normally inhibits evoked transmitter release; this is associated with reduced stimulation-induced net $\mathrm{Ca}^{2+}$ uptake into the synaptic terminal. To explore the possibility that metabolic factors might be involved, nerve ATP levels were reduced by $\geq \mathbf{5 0} \%$ by omitting glucose from the bathing solution. Although quantal content and synaptic delay were unaffected by reduced intracellular ATP, under these conditions $5 \mathrm{~mm}$ exogenous ATP failed to reduce quantal release. Five millimolars of ATP did increase synaptic facilitation, however, which is consistent with a reduced ability to regulate intracellular free $\mathrm{Ca}^{2+}$. Therefore, under conditions of reduced intracellular ATP, the increased resting $\mathrm{Ca}^{2+}$ uptake produced by $5 \mathrm{~mm}$ ATP is not buffered completely. This leads to elevated free $\mathrm{Ca}^{2+}$ levels in the nerve terminal, increasing the amount of transmitter released following an action potential.
\end{abstract}

It is becoming increasingly evident that extracellular ATP is involved in the neuronal regulation of a wide range of excitable tissues (Burnstock, 1985). At the neuromuscular junction, for example, ATP has been postulated as a modulator of transmitter release (Ribeiro, 1979). This is based on the joint observations that stimulation of the motor nerve elicits the release of ATP (Boyd and Forrester, 1968; Silinsky, 1975; Lindgren and Smith, 1986) and that application of exogenous ATP reduces the amount of transmitter secreted in response to stimulation of the motor nerve (Ribeiro and Walker, 1975). At the rat neuromuscular junction, the ATP is apparently released from the presynaptic nerve terminal along with the neurotransmitter acetylcholine (Silinsky, 1975). However, the postsynaptic target cell appears to be the source of the ATP released at the Torpedo electric

\footnotetext{
Received July 25, 1986; revised Dec. 5, 1986; accepted Dec. 15, 1986.

This work was supported by NIH Grants NS13600 and GM07215 (Training Program in Cellular and Molecular Biology). The excellent technical assistance of Michael Chapman is gratefully acknowledged.

Correspondence should be addressed to Dr. Dean O. Smith, Department of Physiology, University of Wisconsin, 1300 University Avenue, Madison, WI 53706.

a Present address: Department of Physiology, Duke University Medical Center, Durham, NC 27710.

Copyright (C) 1987 Society for Neuroscience $0270-6474 / 87 / 051567-07 \$ 02.00 / 0$
}

organ and the crayfish neuromuscular junction (Israel et al., 1976; Lindgren and Smith, 1986). Thus, activation of the motor nerve induces either presynaptic or postsynaptic release of ATP which then may inhibit neurotransmitter secretion.

The mechanism by which exogenous ATP regulates transmitter release is not entirely clear. In a synaptosume-rich preparation from rat cerebral cortex, ATP and adenosine reduce depolarization-induced $\mathrm{Ca}^{2+}$ influx (Ribeiro et al., 1979). Likewise, adenosine has also been shown to inhibit depolarizationinduced $\mathrm{Ca}^{2+}$ uptake in rat brain cortical synaptosomes (Wu et al., 1982; cf. Ribeiro et al., 1979), as well as $\mathrm{Ca}^{2+}$ spikes in hippocampal pyramidal neurons (Proctor and Dunwiddie, 1983). These observations suggest that ATP might modulate transmitter release through an effect on $\mathrm{Ca}^{2+}$ influx into the presynaptic terminal. However, there is also evidence that adenosine does not alter $\mathrm{Ca}^{2+}$ uptake by rat whole-brain synaptosomes (Barr et al., 1985; cf. Dunwiddie, 1985) and that it inhibits transmitter release by a mechanism other than interference with calcium influx in rat hippocampus (Dunwiddie, 1984) and frog neuromuscular junction (Silinsky, 1984). One unexplored possibility is that metabolic factors are involved, since exogenous ATP can be taken up by the presynaptic nerves and incorporated into their metabolic pool (Lindgren and Smith, 1986).

To clarify the relationship between extracellular ATP, transmitter release, and $\mathrm{Ca}^{2+}$ influx, the effects of ATP on quantal content and net calcium uptake into presynaptic nerve terminals were studied at the crayfish neuromuscular junction. Exogenous ATP was found to reduce both transmitter release and ${ }^{45} \mathrm{Ca}^{2+}$ uptake. This effect was independent of ATP's chelation of extracellular $\mathrm{Ca}^{2+}$ but was highly dependent upon the metabolic state of the nerve.

\section{Materials and Methods}

Experimental preparation. All experiments were performed on the efferent nerve innervating the opener muscle of the first walking leg of the adult crayfish (Procambarus clarkii). The inner surface of the muscle was exposed by removing the overlying closer muscle, blood vessels, and connective tissue. The nerve, consisting of 1 excitor and 1 inhibitor axon, could then be clearly seen as it projected along the surface of the muscle (Lindgren and Smith, 1986).

The entire preparation was bathed in saline solution with the following ionic concentrations (mM): $\mathrm{NaCl}, 195 ; \mathrm{KCl}, 5.4 ; \mathrm{CaCl}_{2}, 13.6 ; \mathrm{MgCl}_{2}$ 2.6; D-glucose, 10; HEPES, 10 (pH 7.3). The ATP, ADP, AMP, and adenosine were purchased from Sigma. Following the addition of each of these constituents, the $\mathrm{pH}$ of the saline was adjusted to 7.3 with $\mathrm{NaOH}$. The temperature was maintained at $4^{\circ} \mathrm{C}$ by circulating chilled water around the recording chamber.

ATP is capable of altering free divalent cation concentrations by virtue of its chelating ability. Since transmitter release is highly sensitive to the concentrations of the divalent cations $\mathrm{Ca}^{2+}$ and $\mathrm{Mg}^{2+}$, addition of ATP to the bath would be expected to alter release indirectly by chelating some of the free cations in the bath. Therefore, to distinguish ATP's 
Table 1. Total concentrations of divalent ions in buffered solutions with different concentrations of ATP (pH 7.3)

\begin{tabular}{lllll}
$\begin{array}{l}\text { ATP } \\
(\mathrm{mM})\end{array}$ & $\begin{array}{l}\text { Total } \mathrm{Ca}^{2+} \\
(\mathrm{mM})\end{array}$ & $\begin{array}{l}\text { Free } \mathrm{Ca}^{2+} \\
(\mathrm{mM})\end{array}$ & $\begin{array}{l}\text { Total } \mathrm{Mg}^{2+} \\
(\mathrm{mM})\end{array}$ & $\begin{array}{l}\text { Free } \mathrm{Mg}^{2+} \\
(\mathrm{mM})\end{array}$ \\
\hline 0.5 & 14.00 & 13.61 & 2.70 & 2.60 \\
5.0 & 17.55 & 13.61 & 3.65 & 2.60
\end{tabular}

direct effect on transmitter release from effects mediated through alterations of free cation concentrations in the bath, the extent of chelation was estimated by simultaneously solving the relevant association equations ( $\mathrm{pH} 7.3$ ), using constants of $8500 \mathrm{M}^{-1}$ and $24,500 \mathrm{M}^{-1}$ for the ATP associations with $\mathrm{Ca}^{2+}$ and $\mathrm{Mg}^{2+}$, respectively (Nanninga, 1961). Sufficient $\mathrm{CaCl}_{2}$ and $\mathrm{MgCl}_{2}$ were then added to compensate for chelation. Table 1 presents the divalent cation composition of solutions containing 0.5 and $5.0 \mathrm{~mm}$ ATP corrected for chelation; the remaining ionic constituents were the same as in the normal saline. These estimates were confirmed by measuring the calcium concentration of the bath solution with a calcium-selective electrode. Minor adjustments were then made as necessary and $\mathrm{pH}$ was always corrected to 7.3 with $\mathrm{NaOH}$.

Electrophysiology. The single excitor axon was stimulated in the meropodite region with $0.5 \mathrm{msec}$ pulses using a suction electrode. Synaptic activity was monitored by recording excitatory postsynaptic potentials intracellularly in the opener muscle with 5 to $20 \mathrm{M} \Omega$ microelectrodes filled with $3 \mathrm{M} \mathrm{KCl}$. External recordings of synaptic potentials were obtained by carefully positioning 0.5 to $2 \mathrm{M} \Omega$ microelectrodes filled with $4 \mathrm{M} \mathrm{NaCl}$ over sites on the surface of the muscle fiber where brief, negative potentials were recorded in response to stimulation of the excitor axon (Dudel and Kuffler, 1961). To insure that activity was being recorded from a single synaptic site, the following criteria were used (cf. Niles and Smith, 1982): (1) an action potential in the nerve terminal had to be clearly distinguishable after each stimulus, (2) the rise times of all single quantal potentials had to be less than $1 \mathrm{msec}$, and (3) the amplitudes had to exceed $100 \mu \mathrm{V}$.

These responses included single or multiple quantal potentials or failures of the impulse to release transmitter. At $4^{\circ} \mathrm{C}$, individual quanta appear as distinct quantal potentials or as inflections on the rising phase of a multiple postsynaptic potential (cf. fig. 1, Niles and Smith, 1982). Thus, the number of quanta released in response to an action potential could be counted directly.

These responses were analyzed using methods identical to those described by Niles and Smith (1982). In brief, each response was described by the number of quantal potentials within $20 \mathrm{msec}$ of the nerve terminal action potential, the synaptic delay, and the second quantal latency. Synaptic delays were measured from the first positive peak of the nerve terminal action potential to the onset of the first quantal potential. Second quantal latencies were measured from the first positive peak of the nerve terminal action potential to the first visible onset of the second quantal potential in a multiple response. Whenever more than 1 quantal response to an action potential was recorded, the interquantal latency was calculated by subtracting the synaptic delay from the second quantal latency.

${ }^{45} \mathrm{Ca}^{2+}$ uptake. To measure $\mathrm{Ca}^{2+}$ uptake, the dissected preparation was placed in saline solution containing $\gamma$-methylglutamate $(20 \mathrm{~mm})$ to block activation of the muscle and in ${ }^{45} \mathrm{Ca}^{2+}(13.6 \mathrm{mM}, 14.7 \mathrm{mCi} / \mathrm{mmol})$ for $20 \mathrm{~min}$. The success of these uptake experiments requires thorough removal of extracellular label without significant loss of the intracellular label. Therefore, the preparation was washed in a large volume $(1000$ $\mathrm{ml}$ ) of saline solution $\left(4^{\circ} \mathrm{C}\right)$ containing $5 \mathrm{mM} \mathrm{LaCl}_{3}$ to prevent $\mathrm{Ca}^{2+}$ efflux from the nerve (Van Breeman and De Weer, 1970). The solution was cooled by immersing the container in ice water and was stirred continuously to rinse the tissue thoroughly. The wash solution was periodically assayed for radioactivity to ascertain whether a significant amount of ${ }^{45} \mathrm{Ca}^{2+}$ was accumulating, as this would add to the nerve's ${ }^{45} \mathrm{Ca}^{2+}$ content and thus interfere with the wash. Following a specified period of time, indicated for each experiment, the preparation was rinsed briefly with distilled water to remove salts and then quick-frozen in liquid nitrogen.

To isolate terminal regions of single nerve fibers, the frozen specimens were placed in a lyophilizer and brought to a vacuum before they could thaw (Lindgren and Smith, 1986). The nerve was then dissected from the muscle and weighed on a quartz-fiber "fishpole" balance (Lowry and Passonneau, 1972). It was then placed in a vial containing $50 \mu \mathrm{l}$ of

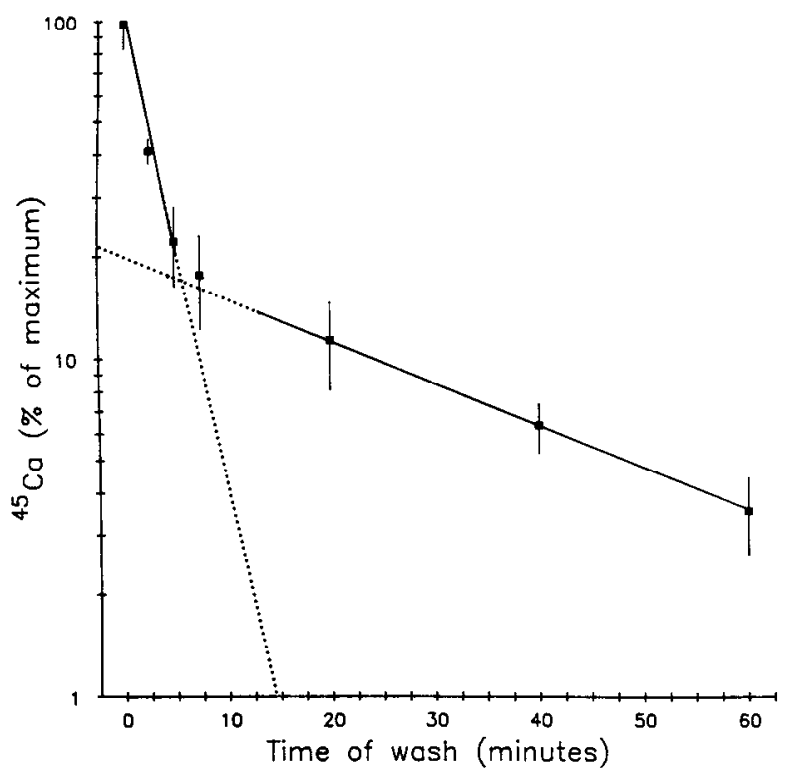

Figure 1. Washout of ${ }^{45} \mathrm{Ca}^{2+}$. The tissue was bathed in saline containing ${ }^{45} \mathrm{Ca}^{2+}$ (sp act, $14.7 \mathrm{Ci} / \mathrm{mol}$ ), rinsed $5 \mathrm{sec}$ in normal saline, and then washed in ice-cold saline containing $5 \mathrm{mM} \mathrm{LaCl}_{3}$ for the times indicated. Each point is the average $(+\mathrm{SE})$ of at least 3 measurements from different preparations. The solid lines were fitted to the data by the method of least squares and the broken lines were extrapolated by eye.

$0.02 \mathrm{~N} \mathrm{HCl}$ and vortexed to dissolve the $\mathrm{Ca}^{2+}$ salts. Ten microliters of $0.1 \mathrm{~N} \mathrm{NaOH}$ were subsequently added to neutralize the $\mathrm{HCl}$ prior to measuring the radioactivity in each vial in a liquid scintillation counter with a program designed for optimal detection of ${ }^{45} \mathrm{Ca}$ and for correction for quenching. Aliquots were also periodically removed from the radioactive bath and assayed for ${ }^{45} \mathrm{Ca}$ to accurately determine the specific activity of the incubation medium. Data are expressed as nanomoles of $\mathrm{Ca}^{2+}$ per milligrams of dry weight of nerve.

The effectiveness of the washout procedure is shown in Figure 1, where the amounts of ${ }^{45} \mathrm{Ca}^{2+}$ are compared to the amounts in the tissue without washing. The removal of ${ }^{45} \mathrm{Ca}^{2+}$ can be seen to be composed of a fast and a slow component. Since each of these components approximates a simple exponential decay, the data were fit to the curve $\mathrm{Ca}(t)=$ $\mathrm{Ca}_{\max } \exp (-t / \tau)$, where $\tau$ is the time constant of the exponential fall of $\mathrm{Ca}(t)$. The fast component, which is thought to reflect the removal of extracellular $\mathrm{Ca}^{2+}$, has a time constant of $3.3 \mathrm{~min}$. The slow component, thought to reflect the leakage of $\mathrm{Ca}^{2+}$ from the cell, has a time constant of $33 \mathrm{~min}$. Thus, in all measurements of net ${ }^{45} \mathrm{Ca}^{2+}$ uptake, the tissue was washed for $20 \mathrm{~min}$, since by this time essentially all (>99\%) of the extracellular $\mathrm{Ca}^{2+}$ will have been removed. In addition, however, $50 \%$ of the intracellular $\mathrm{Ca}^{2+}$ will also have been removed during this period, so subsequent measurements of ${ }^{45} \mathrm{Ca}^{2+}$ uptake will be underestimates.

ATP measurements. Nerve ATP levels were determined using the luciferin-luciferase ("Firefly") method, according to the procedure outlined in Lindgren and Smith (1986). This method allowed quantities of ATP as low as $0.01 \mathrm{pmol}$ to be detected.

\section{Results}

\section{Modulation of evoked transmitter release}

Bath levels of adenosine, AMP, ADP, and ATP were all found to increase following nerve stimulation (Lindgren and Smith, 1986). To determine whether this might play a role in the regulation of synaptic transmission, each was tested for its effect on quantal transmitter release. Transmitter secretion was determined, first, by measuring the average quantal content in normal saline at a single synaptic release site. The compound being tested was then added to the bath solution, and average quantal content was measured again after $15 \mathrm{~min}$. The effect of 


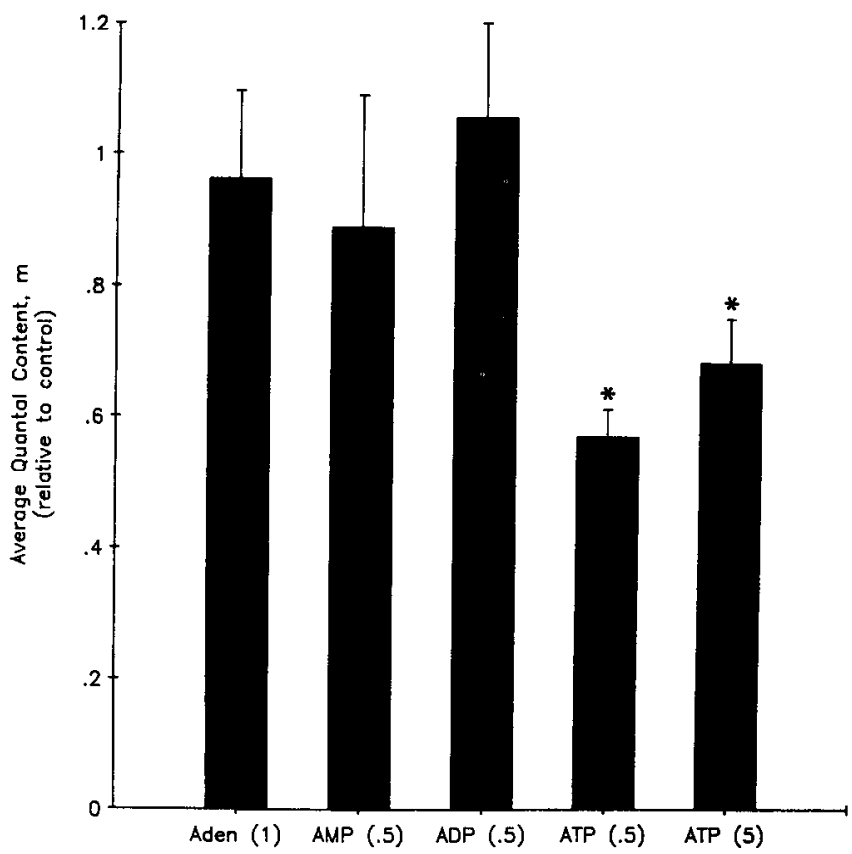

Figure 2. Effect of adenosine and its nucleotides on quantal content. Values represent the average ( \pm SE) quantal content measured in the presence of the indicated compounds as compared to control values measured in normal saline. The numbers in parentheses indicate the concentration (in $\mathrm{mm}$ ) of the compound in the bath. A total of 26 preparations are represented, with at least 3 preparations represented per compound. Asterisks indicate that the values are statistically different from 1 ( 0.025 level, 2-tailed $t$ test).

the compound on quantal content was calculated by taking the ratio of average quantal content after addition of the compound to the average quantal content in normal saline.

As shown in Figure 2, only ATP was found to have a significant effect on quantal content; 0.5 and $5 \mathrm{~mm}$ ATP inhibited release by 43 and $31 \%$, respectively. These reductions were statistically significant at the 0.025 level (2-tailed $t$ test). The inhibition caused by $0.5 \mathrm{~mm}$ ATP could always be reversed with washing. In contrast, the effects of $5 \mathrm{mM}$ ATP, which occurred more rapidly, were seldom reversed after up to $2 \mathrm{hr}$ of washing. These effects are illustrated in Figure 3.

Neither ADP, AMP, nor adenosine had a significant effect on quantal content. Furthermore, spontaneous quantal release (seen as miniature endplate potentials) was not affected by any of the agents tested. Thus, ATP (but not adenosine) depresses evoked quantal transmitter release in this preparation (cf. Silinsky and Ginsborg, 1983).

These results might be expected if elevated extracellular ATP were found to cause action potential conduction block in the terminal arborization. This possibility was tested by examining the extracellular records of transmitter release failure for the presence of nerve terminal action potentials (cf. Niles and Smith, 1982). However, there was no evidence of systematic conduction failure associated with elevated extracellular ATP. Although conduction failure may have occurred distal to the recording site, this possibility seems unlikely.

\section{Effect of ATP on presynaptic Ca uptake}

A reduction in the amount of $\mathrm{Ca}^{2+}$ influx into the synaptic terminal could explain ATP's effect on transmitter release. Therefore, stimulation-induced ${ }^{45} \mathrm{Ca}^{2+}$ uptake in this prepara-

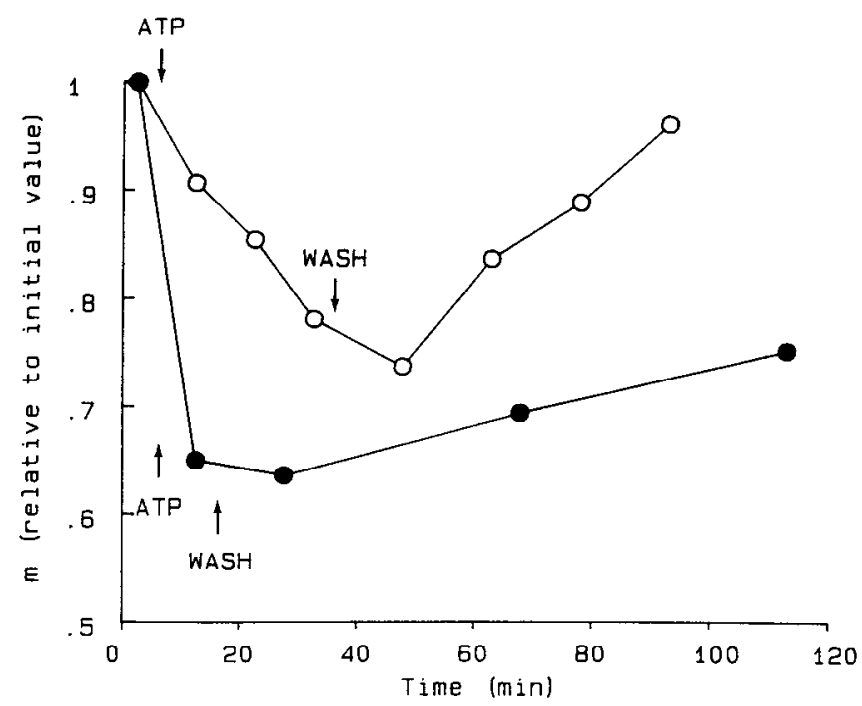

Figure 3. Effect of ATP on quantal content. The average number of quanta, $m$, released during stimulation at $2 \mathrm{~Hz}$ are shown for 2 preparations, one exposed to $0.5 \mathrm{~mm}$ ATP (open circles), the other to $5 \mathrm{~mm}$ ATP (filled circles). The perfusate was switched to saline containing ATP, then back to normal saline at the times indicated by the arrows. Each point is the average of between 250 and 500 consecutive synaptic responses.

tion was measured in the presence and the absence of ATP. As shown in Table 2, stimulation increased uptake by $66 \%$. This corresponds to the extra uptake of $0.18 \mathrm{pmol} / \mathrm{mg}$ dry weight per impulse.

Inclusion of $0.5 \mathrm{~mm}$ ATP in the incubation medium had no effect on resting ${ }^{45} \mathrm{Ca}^{2+}$ uptake, but it prevented the stimulationinduced increase. In Table 2, this inhibition of net ${ }^{45} \mathrm{Ca}^{2+}$ uptake is evidenced as a significant reduction in the difference between ${ }^{45} \mathrm{Ca}^{2+}$ uptake in stimulated and nonstimulated preparations. Thus, $0.5 \mathrm{~mm}$ ATP inhibits net stimulation-induced $\mathrm{Ca}^{2+}$ influx into the nerve. This effect depends on the external ATP levels, though. Measurement of net ${ }^{45} \mathrm{Ca}^{2+}$ uptake in preparations exposed to $5 \mathrm{~mm}$ ATP revealed a $650 \%$ increase in ${ }^{45} \mathrm{Ca}^{2+}$ accumulation in nonstimulated, as compared to control, preparations (Table 2). Stimulation had no further effect on ${ }^{45} \mathrm{Ca}^{2+}$ uptake.

Table 2. Effect of ATP on stimulation-induced net ${ }^{45} \mathrm{Ca}$ influx

Net ${ }^{45} \mathrm{Ca}$ uptake (nmol/mg dry wt)

\begin{tabular}{llll} 
& \multicolumn{3}{l}{ Net ${ }^{45} \mathrm{Ca}$ uptakc $(\mathrm{nmol} / \mathrm{mg}$ dry wt) } \\
\cline { 2 - 4 } Experimental & $\begin{array}{l}\text { Nonstimu- } \\
\text { lated }\end{array}$ & Stimulated & Difference \\
\hline Control & $4.27 \pm 0.76^{a}$ & $7.08 \pm 0.65^{a}$ & $2.81 \pm 1.01^{b}$ \\
& $\begin{array}{l}(n=12) \\
(n=13)\end{array}$ & \\
ATP $(0.5 \mathrm{mM})$ & $4.49 \pm 0.53$ & $4.97 \pm 0.47$ & $0.48 \pm 0.71^{b}$ \\
& $(n=15)$ & $(n=11)$ & \\
ATP $(5 \mathrm{mM})$ & $32.1 \pm 6.9$ & $\begin{array}{l}\text { 29.3 } \pm 6.1 \\
(n=6)\end{array}$ & $-2.8 \pm 7.2$ \\
& $(n=7)$ & \\
\hline
\end{tabular}

Average $( \pm \mathrm{SE})$ values of ${ }^{45} \mathrm{Ca}$ uptake obtained from preparations incubated with $13.6 \mathrm{mM}{ }^{45} \mathrm{Ca}$ (sp act, $14.7 \mathrm{Ci} / \mathrm{mol}$ ) in either normal saline containing $20 \mathrm{~mm}$ $\gamma$-methylglutamate (Control) or in saline containing ATP as well as $\gamma$-methylglutamate (ATP). The nerves were incubated for $20 \mathrm{~min}$, either at rest or during stimulation $(10 \mathrm{~Hz})$, and then washed for $20 \mathrm{~min}$ in cold saline containing $5 \mathrm{~mm}$ $\mathrm{LaCl}_{3}$. The number of preparations $(n)$ is shown in parentheses.

a Significantly different at the 0.05 level.

${ }^{\mathrm{b}}$ Significantly different at the 0.05 level. 


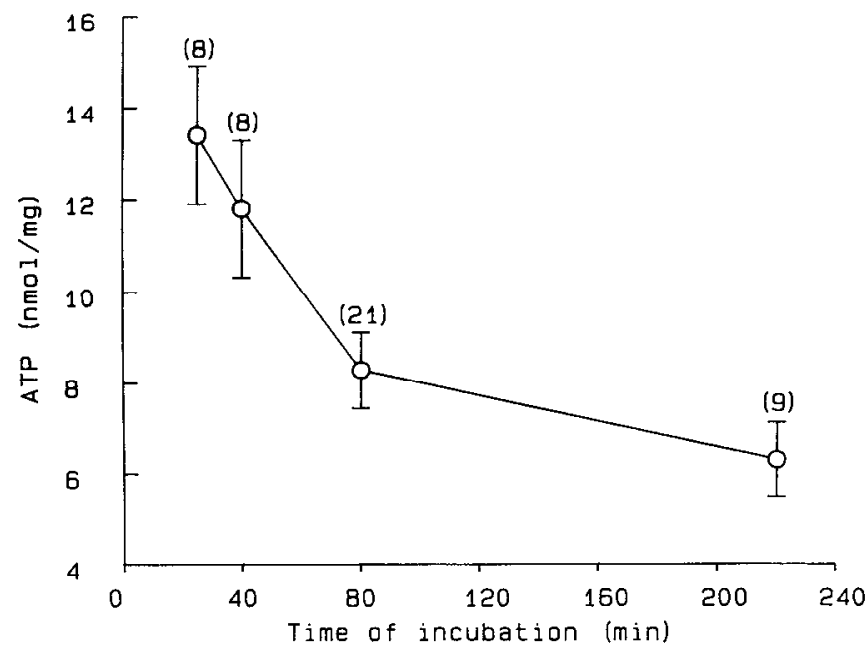

Figure 4. Nerve ATP levels in preparations incubated in glucose-free saline. The tissue was incubated for the times indicated and then quickfrozen in liquid nitrogen for subsequent measurement of ATP. The average $( \pm \mathrm{SE})$ values at each of the incubation times are presented; the number of nerves analyzed is given in parentheses.

Therefore, exposure to high concentrations of ATP ( $5 \mathrm{~mm}$ ) promotes the net accumulation of $\mathrm{Ca}^{2+}$ through a process that is independent of nerve stimulation.

Stimulation-induced $\mathrm{Ca}^{2+}$ uptake also appears to be inhibited by $5 \mathrm{~mm}$ ATP, although the increased resting $\mathrm{Ca}^{2+}$ uptake obscures the effect. The ATP-induced resting permeability increase may result, however, in a higher free $\mathrm{Ca}^{2+}$ concentration in the nerve terminal, opposing the inhibitory effect of ATP on stimulation-induced $\mathrm{Ca}^{2+}$ entry. This would indicate that higher concentrations of ATP (5 mM) may not necessarily reduce the average number of quanta $(\mathrm{m})$ more effectively than do lower concentrations $(0.5 \mathrm{~mm})$, which is exactly what we observed (Fig. 2).

\section{Metabolic dependence of ATP's modulatory role}

Because intracellular ATP has such a critical role in cell metabolism, and extracellular ATP has been shown to enter the nerve cell and participate in an ATP-dependent reaction (Lindgren and Smith, 1986), the relationship between the energy state of the cell and the ability of exogenous ATP to modulate transmitter release was examined. Experiments were designed to reduce intracellular ATP levels and then to assess quantal release in both normal and ATP-containing saline.

\section{Alteration of intracellular ATP levels}

To carry out these experiments, intracellular ATP levels needed to be reduced without dramatically perturbing cellular homeo-

\section{Table 3. Effect of glucose on time course of release}

\begin{tabular}{llll} 
Condition & $m$ & $\begin{array}{l}\text { Synaptic delay } \\
\text { (mscc) }\end{array}$ & $\begin{array}{l}\text { Interquantal } \\
\text { latency } \\
(\mathrm{msec})\end{array}$ \\
\hline+ Glucose & $0.39 \pm 0.06$ & $5.74 \pm 0.21$ & $2.55 \pm 0.22$ \\
- Glucose & $0.36 \pm 0.05$ & $5.84 \pm 0.17$ & $2.70 \pm 0.17$
\end{tabular}

Average ( \pm SE) values of data obtained from 14 preparations in which glucose $(10$ $\mathrm{mM}$ ) was present in the incubation medium and from 14 preparations in which glucose was absent. The responses to approximately 500 action potentials, occurring at a rate of 2 impulses/sec, were measured in each preparation.

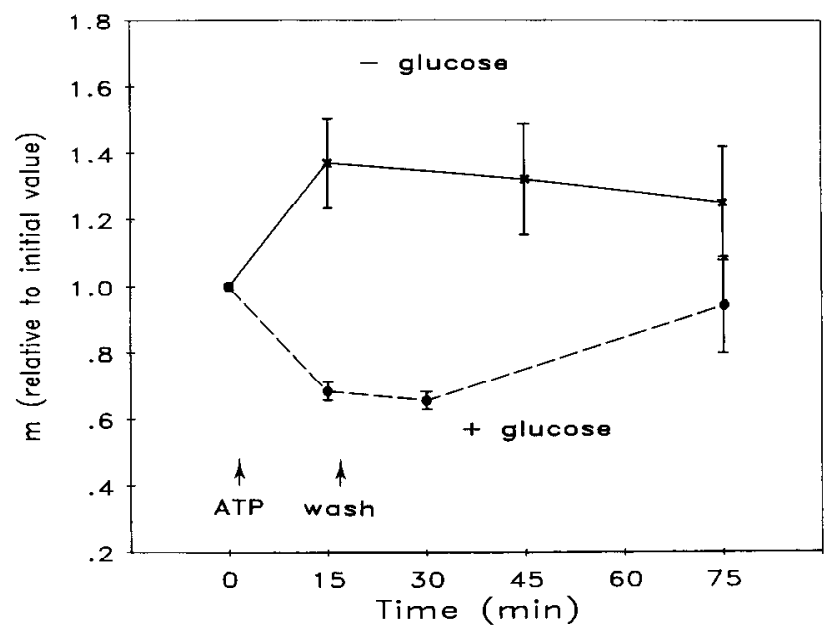

Figure 5. Glucose dependence of ATP's effect on quantal content. At each of 4 sites, $m$ was first measured in normal saline (+ glucose; dashed line) and then after the addition of ATP for $15 \mathrm{~min}$, followed by intermittent measurements during wash with normal saline (+ glucose; dashed line). $m$ was measured similarly at 4 additional sites after incubating the preparations for at least $80 \mathrm{~min}$ in glucose-free saline (- glucose; solid line). Each point represents the average response to at least 500 action potentials and is expressed relative to the initial quantal content.

stasis. Exposure to a mitochondrial inhibitor, such as 2,4-dinitrophenol, was judged unsuitable for this purpose because it leads to irreversible changes in transmitter release (Atwood et al., 1972), making it impossible to draw inferences about the modulation of release by extracellular ATP. However, an effective method for reducing intracellular ATP, which had little direct effect on transmitter release, proved to be the elimination of glucose from the bathing medium. This is illustrated in Figure 4, which plots nerve ATP levels after various periods of incubation in glucose-free saline. After $80 \mathrm{~min}$, nerve ATP declined by approximately $50 \%$. This rapid decrease was followed by a slower reduction in ATP over the next $2 \mathrm{hr}$. Thus, the nerve cell is incapable of maintaining normal ATP levels in the absence of glucose. Subsequent experiments were performed under these conditions.

[There does, however, appear to be a small store of endogenous substrate available, since addition of oligomycin $(5 \mu \mathrm{g} / \mathrm{ml})$, an inhibitor of mitochondrial ATP production, increased the rate of fall in ATP levels. Specifically, they fell to $8.3 \pm 0.8$ $\mathrm{nmol} / \mathrm{mg}$ dry weight $( \pm \mathrm{SE}, n=21)$ after $80 \mathrm{~min}$ of incubation in glucose-free saline; incubation for the same length of time in glucose-free saline containing oligomycin caused ATP levels to fall to $6.3 \pm 0.7 \mathrm{nmol} / \mathrm{mg}$ dry weight $(n=20)$, an additional 2 $\mathrm{nmol} / \mathrm{mg}$ dry weight. This represents a statistically significant effect of the oligomycin on ATP levels ( 0.05 level; 2 -tailed $t$ test).]

\section{Modulation of transmitter release}

Reduced intracellular ATP levels did not demonstrably affect transmitter release. As shown by data summarized in Table 3, neither average quantal content, synaptic delay, nor interquantal latency were significantly altered when glucose was omitted from the bath. This indicates that the magnitude and the time course of evoked transmitter release were not altered by the associated decline in nerve ATP.

The effect of exogenous ATP on transmitter release in cells with lowered intracellular ATP was examined. Quantal content 


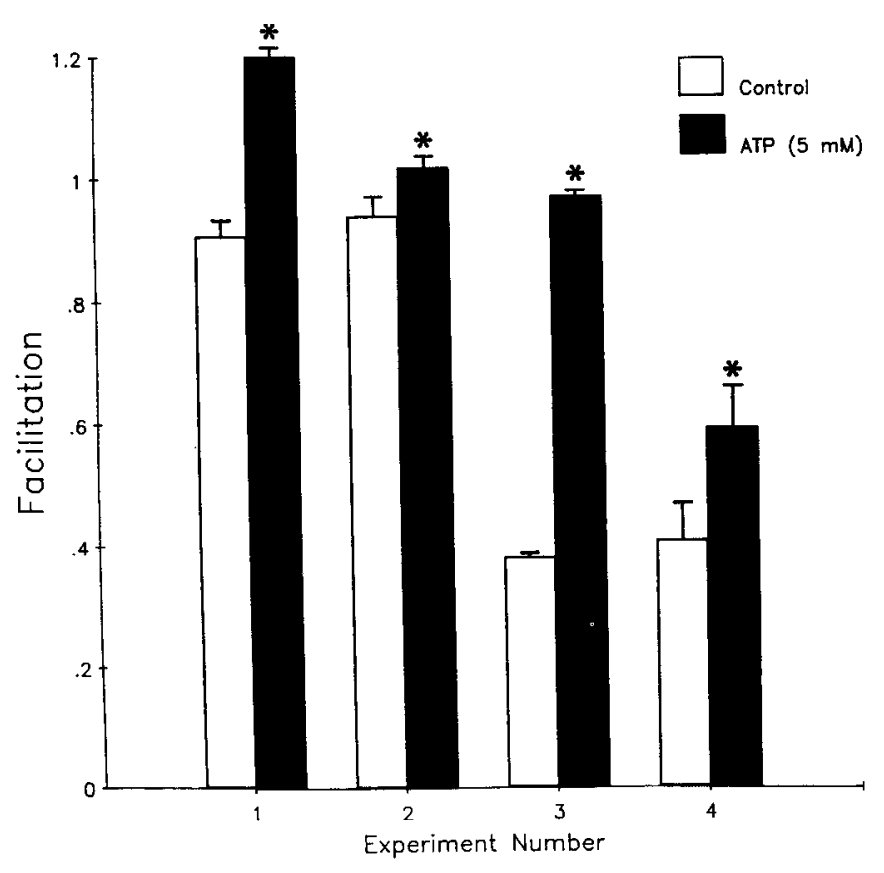

Figure 6. Effect of $5 \mathrm{~mm}$ ATP on synaptic facilitation in preparations with reduced intracellular ATP. At each of 4 sites, the extent of synaptic facilitation was determined in control saline and again after the addition of $5 \mathrm{mM}$ ATP. Valucs represent the averages $( \pm \mathrm{SE})$ of $250-500$ paired pulses, delivered at a frequency of $0.5 \mathrm{~Hz}$. Synaptic facilitation was defined as $\left(m_{2}-m_{1}\right) / m_{1}$, where $m_{1}$ and $m_{2}$ refer to the average number of quanta of transmitter released by the first and second pulses, respectively. Asterisks indicate that the amount of facilitation in the presence of $5 \mathrm{~mm}$ ATP is statistically different from the amount of facilitation in control saline ( 0.05 level, 2-tailed $t$ test).

was depressed by $0.5 \mathrm{~mm}$ ATP, as in preparations bathed in glucose-containing medium (Fig. 2). By contrast, quantal content was not decreased by $5 \mathrm{~mm}$ ATP in glucose-deprived tissue. Indeed, as shown in Figure 5, there was a marginal, persistent increase in quantal content under these conditions.

In summary, these data suggest that the effect of $5 \mathrm{~mm}$ exogenous ATP on transmitter release in preparations with reduced intracellular $\Lambda \mathrm{TP}$ might result from a combination of increased resting $\mathrm{Ca}^{2+}$ influx (Table 2 ) and possibly less active clearance of $\mathrm{Ca}^{2+}$ from transmitter release sites in the nerve terminal. This may result in an elevation of free $\mathrm{Ca}^{2+}$ levels, which would lead to the observed potentiation of evoked transmitter release.

\section{Synaptic facilitation}

If addition of $5 \mathrm{~mm}$ ATP to preparations with reduced intracellular ATP levels indeed reduces $\mathrm{Ca}^{2+}$ clearance from nerve terminals, synaptic facilitation should also be more pronounced, since it is presumably due to residual calcium remaining in the terminal following an initial impulse (Katz and Miledi, 1968; Zucker, 1974). This possibility was tested; the results are presented in Figure 6. Specifically, $5 \mathrm{~mm}$ ATP increased synaptic facilitation in preparations with reduced intracellular ATP levels. It seems quite likely, therefore, that reducing intracellular ATP compromises the ability of the nerve terminal to exclude and/ or sequester free $\mathrm{Ca}^{2+}$. Thus, the increased resting $\mathrm{Ca}^{2+}$ levels due to exposure to $5 \mathrm{~mm}$ ATP (Table 2) are caused, at least in part, by a decreased ability to extrude $\mathrm{Ca}^{2+}$.

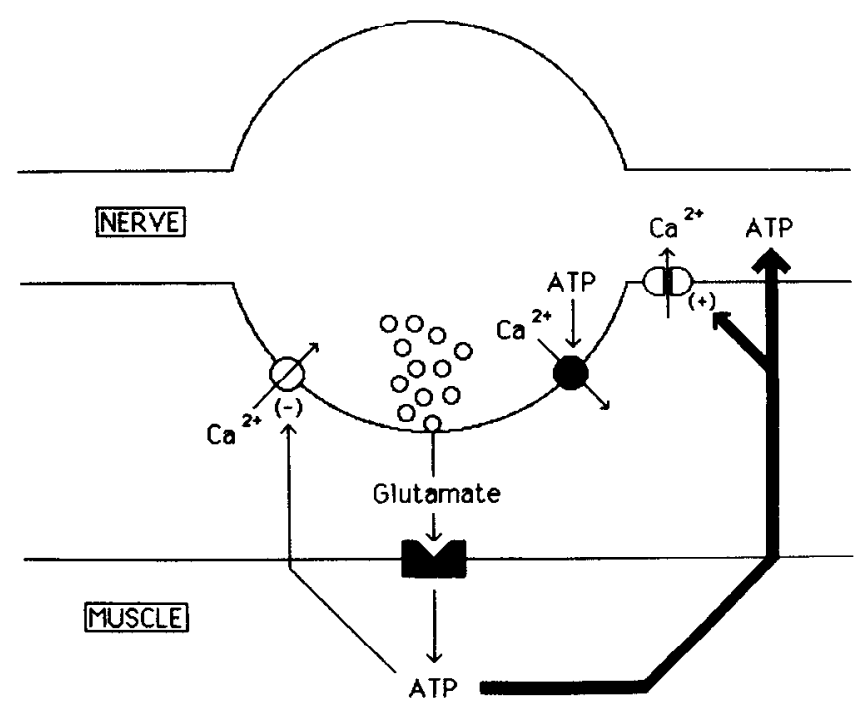

Figure 7. Summary of ATP's effects on the nerve terminal. The release of ATP from the muscle depicted with the thin line represents effects observed following application of $0.5 \mathrm{~mm}$ ATP; the thick line represents effects obtained with application of $5 \mathrm{~mm}$ ATP.

\section{Discussion}

In this study, exogenous ATP was found to reduce quantal transmitter release at the excitatory neuromuscular junction in the walking leg of the crayfish. Similar results have been obtained by others at the neuromuscular junctions of the rat and frog (Ribeiro and Walker, 1975), the longitudinal muscle strip of guinea pig ileum, and in slices of rat cerebral cortex (Vizi and Knoll, 1976), Torpedo electric organ (Israel et al., 1980), and from preganglionic frog nerves (Silinsky and Ginsborg, 1983). This study is unique, however, because this effect of ATP was shown to depend on the presence of glucose in the bathing medium. In the absence of glucose, under conditions in which intracellular ATP is reduced by approximately 50\%, exogenous ATP has a dual effect: $0.5 \mathrm{~mm}$ ATP inhibits release, whereas 5 mM ATP is facilitatory. In some cases, the latter effect is very large and prolonged.

These observations on transmitter release are paralleled by a similar, although not identical, dual effect of ATP on ${ }^{45} \mathrm{Ca}^{2+}$ uptake into regions of the motor nerve near the synaptic terminals. Five millimolars of ATP increased the resting uptake of ${ }^{45} \mathrm{Ca}^{2+}$; however, both 0.5 and $5 \mathrm{~mm}$ ATP reduced stimulation-induced uptake. Likewise, in a synaptosome-rich preparation from rat cerebral cortex depolarized by high potassium, $0.2 \mathrm{mM}$ ATP decreased the uptake of ${ }^{45} \mathrm{Ca}^{2+}, 1 \mathrm{~mm}$ ATP had no effect, and 2 mм ATP increased it (Ribeiro et al., 1979). Furthermore, it has been shown that 2 mM ATP increases the influx of ${ }^{45} \mathrm{Ca}^{2+}$ into isolated mast cells (Dahlquist, 1974) and Ehrlichascites tumor cells (Landry and Lehninger, 1976), and that 1 mM ATP increases ${ }^{45} \mathrm{Ca}^{2+}$ influx into Friend virus-infected murine erythroleukemia cells (Chahwala and Cantley, 1984).

Since $\mathrm{Ca}^{2+}$ is required for transmitter release (Katz and Miledi, 1967), the corresponding effects of ATP on transmitter release and on net ${ }^{45} \mathrm{Ca}^{2+}$ uptake suggest that extracellular ATP reduces the release of transmitter from crayfish excitatory motor nerve terminals by reducing the influx of $\mathrm{Ca}^{2+}$ in response to an action potential. However, an increased efflux of $\mathrm{Ca}^{2+}$ due to ATP would also be consistent with our data. In addition, high concentrations of ATP increase the net uptake of ${ }^{45} \mathrm{Ca}^{2+}$ at rest. The 
predicted influence of such an effect on transmitter release is not immediately apparent. Measurement of ${ }^{45} \mathrm{Ca}^{2+}$ uptake into a tissue gives only a measure of the net accumulation of $\mathrm{Ca}^{2+}$ in the cell. It indicates neither the intracellular location of the $\mathrm{Ca}^{2+}$ nor its disposition within the cell. However, it does seem probable that the $\mathrm{Ca}^{2+}$ permeability induced by $5 \mathrm{~mm}$ ATP is not localized to transmitter release sites because the large resting permeability increase produced by $5 \mathrm{~mm}$ ATP had a negligible influence on transmitter release in preparations with normal intracellular ATP levels.

In contrast, $5 \mathrm{~mm}$ ATP increased transmitter release in preparations with a reduced intracellular ATP concentration. This could have been caused by less effective $\mathrm{Ca}^{2+}$ buffering in the nerve because of lower ATP levels. If this were the case, the resting $\mathrm{Ca}^{2+}$ influx induced by $5 \mathrm{~mm}$ ATP could lead to a sizable elevation of free $\mathrm{Ca}^{2+}$ levels in the terminal, resulting in increased transmitter release. The observed increase in synaptic facilitation in low-ATP preparations upon exposure to $5 \mathrm{~mm}$ ATP is consistent with this interpretation.

A summary of these effects and their relationship to one another is diagrammed in Figure 7 . The interaction of the excitatory transmitter glutamate with its receptors causes ATP to be released, probably from the muscle, into the extracellular space (Lindgren and Smith, 1986). Two possible presynaptic effects of the released ATP are shown: (1) Exogenous ATP inhibits the stimulation-induced entry of $\mathrm{Ca}^{2+}$ into the terminal, reducing the amount of transmitter released in response to an action potential; (2) high concentrations of ATP also increase a nonspecific resting influx of $\mathrm{Ca}^{2+}$ into the nerve. In preparations with normal intracellular ATP levels, this resting influx is buffcred in the terminal sufficiently for the inhibitory cffect of cxogenous ATP on stimulation-induced release to predominate. However, in preparations with reduced intracellular ATP levels, the resting influx of $\mathrm{Ca}^{2+}$ predominates, leading to an increased release of transmitter.

The relative contributions of these 2 competing effects under physiological conditions depend on whether intracellular ATP levels ever become as low as the preparations in this study, and on whether extracellular ATP levels ever get as high as $5 \mathrm{~mm}$. Wide variations in blood glucose, associated with molting, environmental stress, and oxygen depletion, have been reported (Telford, 1974, 1975). Since these measurements are probably a reflection of alterations in metabolism, it is possible that intracellular ATP levels may become significantly reduced under these conditions. Whether extracellular ATP levels ever get high enough to effect a generalized $\mathrm{Ca}^{2+}$ permeability increase is less ccrtain. Indirect measurcments indicate that the ATP in the space immediately surrounding the nerve may rise to millimolar concentrations following high-frequency stimulation (Lindgren and Smith, 1986). Nonetheless, direct evidence is lacking.

The mechanism whereby ATP increases the net uptake of $\mathrm{Ca}^{2+}$ into the nerve cell needs further clarification, since it is such an unusual occurrence. One possibility is that exogenous ATP renders the cell membrane permeable both to ATP (Lindgren and Smith, 1986) and to $\mathrm{Ca}^{2+}$ by the same mechanism. The ATP that enters the cell may then increase $\mathrm{Ca}^{2+}$ entry further by stimulating $\mathrm{Ca}^{2+}$ uptake into intracellular organelles. Such a mechanism was suggested by Landry and Lehninger (1976) for the ATP-stimulated ${ }^{45} \mathrm{Ca}^{2+}$ uptake into Ehrlich-ascites tumor cells, since the uptake of $\mathrm{Ca}^{2+}$ was prevented by oligomycin. Whatever the mechanism proves to be, the fact that exogenous ATP induces similar permeability changes in a variety of cell types and species suggests that it may be a fundamental property of all cells. It is particularly intriguing that transformed cells are more sensitive to exogenous ATP than nontransformed cells (cf. Landry and Lehninger, 1976; Rozengurt and Heppel, 1979; De and Weisman, 1984). Thus, insight gained into the molecular mechanisms responsible for this phenomenon may not only give insight into normal cellular function, but may also suggest fundamental differences between normal and transformed cells.

\section{References}

Atwood, H. L., F. Lang, and W. A. Morin (1972) Synaptic vesicles: Selective depletion in crayfish excitatory and inhibitory axons. Science 176: 1353-1355.

Barr, E., L. C. Daniell, and S. W. Leslie (1985) Synaptosomal calcium uptake unaltered by adenosine and 2-chloroadenosine. Biochem. Pharmacol. 34: 713-715.

Boyd, I. A., and T. Forrester (1968) The release of adenosine triphosphate from frog skeletal muscle in vitro. J. Physiol. (Lond.) 199: 115-135.

Burnstock, G. (1985) Purinergic mechanisms broaden their sphere of influence. Trends Neurosci. 8: 5-6.

Chahwala, S. B., and L. C. Cantley (1984) Extracellular ATP induces ion fluxes and inhibits growth of Friend erythroleukemia cells. J. Biol. Chem. 259: 13717-13722.

Dahlquist, R. (1974) Determination of ATP-induced ${ }^{45}$ calcium uptake in rat mast cells. Acta Pharmacol. Toxicol. 35: 1-10.

De, B. K., and G. A. Weisman (1984) The role of calcium ions in the permeability changes produced by external ATP in transformed 3T3 cells. Biochim. Biophys. Acta 775: 381-388.

Dudel, J., and S. W. Kuffler (1961) The quantal nature of transmission and spontaneous miniature potentials at the crayfish neuromuscular junction. J. Physiol. (Lond.) 155: 514-529.

Dunwiddie, T. V. (1984) Interactions between the effects of adenosine and calcium on synaptic responses in rat hippocampus in vivo. $\mathrm{J}$. Physiol. (Lond.) 350: 545-559.

Dunwiddie, T. V. (1985) The physiological role of adenosine in the central nervous system. Int. Rev. Neurobiol. 27: 63-139.

Israel, M., B. Lesbats, F. M. Meunier, and J. Stinnakre (1976) Postsynaptic release of adenosine triphosphate induced by single impulse transmitter action. Proc. R. Soc. Lond. [Biol.] 193: 461-468.

Israel, M., B. Lesbats, R. Manaranche, F. M. Meunier, and P. Frachon (1980) Retrograde inhibition of transmitter release by ATP. J. Neurochem. 34: 923-932.

Katz, B., and R. Miledi (1967) The release of acetylcholine from nerve endings by graded electric pulses. Proc. R. Soc. Lond. [Biol.] 167: 2338.

Katz, B., and R. Miledi (1968) The role of calcium in neuromuscular facilitation. J. Physiol. (Lond.) 195: 481-492.

Landry, Y., and A. L. Lehninger (1976) Transport of calcium ions by Ehrlich ascites-tumour cells. Biochem. J. 158: 427-438.

Lindgren, C. A., and D. O. Smith (1986) Increased presynaptic ATP levels coupled to synaptic activity at the crayfish neuromuscular junction. J. Neurosci. 6: 2644-2652.

Lowry, O., and J. V. Passonneau (1972) A Flexible System of Enzymatic Analysis, pp. 223-235, Academic, New York.

Nanninga, L. B. (1961) The association constant of the complexes of adenosine triphosphate with magnesium, calcium, strontium, and barium ions. Biochim. Biophys. Acta 54: 330-338.

Niles, W., and D. O. Smith (1982) Effects of hypertonic solutions on quantal transmitter release at the crayfish neuromuscular junction. J. Physiol. (Lond.) 329: 185-202.

Proctor, W. R., and T. V. Dunwiddic (1983) Adenosine inhibits calcium spikes in hippocampal pyramidal neurons in vitro. Neurosci. Lett. 35: 197-201.

Ribeiro, J. A. (1979) Purinergic modulation of transmitter release. J. Theoret. Biol. 80: 259-270.

Ribeiro, J. A., and J. Walker (1975) The effects of adenosine triphosphate and adenosine diphosphate on transmission at the rat and frog neuromuscular junctions. Br. J. Pharmacol. 54: 213-218.

Ribeiro, J. A., A. M. Sa-Almeida, and J. M. Namorado (1979) Adenosine and adenosine triphosphate decrease ${ }^{45} \mathrm{Ca}$ uptake by synaptosomes stimulated by potassium. Biochem. Pharmacol. 28: 1297-1300. 
Rozengurt, E., and L. A. Heppel (1979) Reciprocal control of membrane permeability of transformed cultures of mouse cell lines by external and internal ATP. J. Biol. Chem. 254: 708-714.

Silinsky, E. M. (1975) On the association between transmitter secretion and the release of adenine nucleotides from mammalian motor nerve terminals. J. Physiol. (Lond.) 247: 145-162.

Silinsky, E. M. (1984) On the mechanism by which adenosine receptor activation inhibits the release of acetylcholine from motor nerve endings. J. Physiol. (Lond.) 346: 243-256.

Silinsky, E. M., and B. L. Ginsborg (1983) Inhibition of acetylcholine release from preganglionic frog nerves by ATP but not adenosine. Nature 305: 327-328.

Telford, M. (1974) Blood glucose in crayfish-I. Variations associated with molting. Comp. Biochem. Physiol. 47 A: 461-468.
Telford, M. (1975) Blood glucose in crayfish-II. The source of glucose and role of the eyestalk factor in hyperglycemia of Cambarus robustus. Comp. Biochem. Physiol. 51B: 69-73.

Van Breeman, C., and P. De Weer (1970) Lanthanum inhibition of ${ }^{45} \mathrm{Ca}$ efflux from the squid giant axon. Nature 226: 760-761.

Vizi, E. S., and J. Knoll (1976) The inhibitory effect of adenosine and related nucleotides on the release of acetylcholine. Neuroscience 1 : 391-398.

Wu, P. H., J. W. Phillis, and D. L. Thierry (1982) Adenosine receptor agonists inhibit $\mathrm{K}^{+}$-evoked $\mathrm{Ca}^{2+}$ uptake by rat brain cortical synaptosomes. J. Neurochem. 39: 700-708.

Zucker, R. S. (1974) Characteristics of crayfish neuromuscular facilitation and their calcium dependence. J. Physiol. (Lond.) 241: 91110. 